\title{
HYDRODYNAMIC MODELS OF THE ACCRETION STREAM IN MAGNETIC CATACLYSMIC VARIABLES
}

\author{
Kunegunda E. Belle ${ }^{1}$
}

Here I use the MHD code, FLASH, from the University of Chicago ASCI Center for Astrophysical Thermonuclear Flashes, to simulate initial two-dimensional simple models of accretion streams in magnetic CVs. These models explore the evolution of inflowing material for two white dwarf masses; $0.5 M_{\odot}$ and $0.9 M_{\odot}$. It is seen that a discontinuity forms at a height of $6.1 \times 10^{8} \mathrm{~cm}$ above the white dwarf 'surface' for $M_{\mathrm{WD}}=0.5 M_{\odot}$ and at $1.6 \times 10^{9}$ $\mathrm{cm}$ for $M_{\mathrm{WD}}=0.9 M_{\odot}$. These models will be developed further with the ultimate goal of completing a three-dimensional model of magnetic accretion onto a WD surface.

The Modcls: Models using different white dwarf masses were constructed. For each model, a 2D cartesian grid of width $4 \times 10^{7} \mathrm{~cm}$ and height $5 \times 10^{9}$ $\mathrm{cm}$ was used. An accretion stream of this width roughly corresponds to a fractional accretion area of $f=0.001$ on the surface of a $0.9 M_{\odot}$ white dwarf. The computational grid is set up so that one computational block is $2 \times 10^{7} \mathrm{~cm}$ on a side and three levels of adaptive mesh refinement (AMR) are allowed during the computation. Protons and electrons are used for the stream composition, the material is allowed to radiate, and a gamma-law EOS and plane parallel gravity are ('mployed. The entire computational volume is given the initial stream parameters $T=50,000 \mathrm{~K}$ and $\rho=1 \times 10^{-10} \mathrm{~g} \mathrm{~cm}^{-3}$. A downward velocity of $-3 \times 10^{5} \mathrm{~cm} \mathrm{~s}^{-1}$ is given to material above $y=3 \times 10^{9} \mathrm{~cm}$. Each model uses a $1 \mathrm{MG}$ magnetic field.

Model 1. This simulation was performed for $M_{\mathrm{WD}}=0.5 M .(\log g=7.86)$. Figure 1 displays the temperature profile at times $t=0$ and $t=69$ $\mathrm{s}$. This is the time at which a discontinuity forms at $y=6.1 \times 10^{8} \mathrm{~cm}$, as can be seen in the plot. After time $t=69 \mathrm{~s}$, a numerically seeded instability develops.

Model 2. This simulation was performed for $M_{\mathrm{WD}}=0.9 M_{\odot}(\log g=8.49)$. Figure 2 displays the temperature profile at times $t=0$ and $t=42.5$ $\mathrm{s}$. This is the time at which a discontinuity forms at

\footnotetext{
${ }^{I}$ X-2 MS P225, Los Alamos National Laboratory, Los Alamos, NM 87545 (belle@lanl.gov).
}

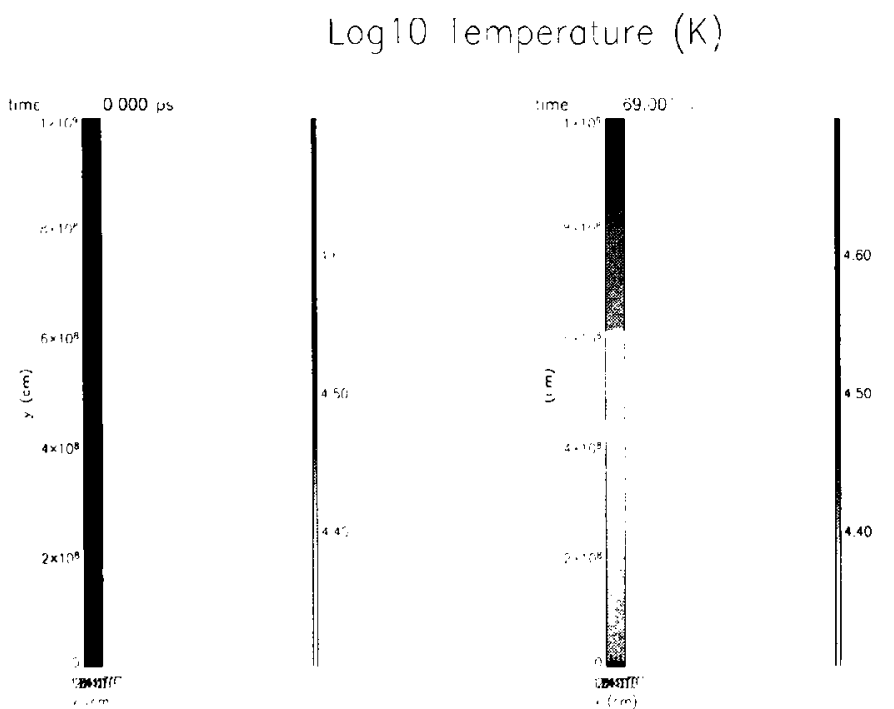

Fig. 1. Temperature profile for Model $1\left(M_{\mathrm{WD}}=\right.$ $\left.0.5 M_{\odot}\right)$.

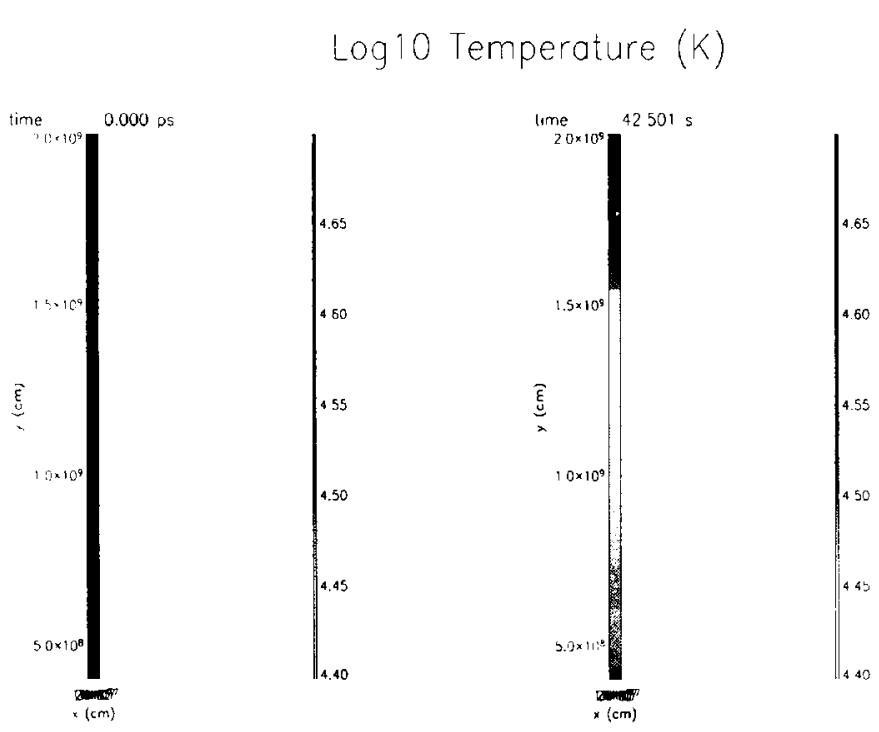

Fig. 2. Temperature profile for Model $2\left(M_{\mathrm{WD}}=\right.$ $\left.0.9 M_{\odot}\right)$.

$y=1.6 \times 10^{9} \mathrm{~cm}$, as can be seen in the plot. After time $t=42 \mathrm{~s}$, a numerically seeded instability develops.

The software used in this work was in part developed by the DOE-supported ASCI/Alliance Center for Astrophysical Thermonuclear Flashes at the University of Chicago. 